\title{
A Propensity Score Analysis of the Impact of Invasive Intracranial Pressure Monitoring on Outcomes after Severe Traumatic Brain Injury
}

\author{
Deepak Agrawal, ${ }^{1}$ Krishnan Raghavendran, ${ }^{2}$ Douglas E. Schaubel, ${ }^{3}$ \\ Mahesh C. Mishra, ${ }^{4}$ and Venkatakrishna Rajajee ${ }^{5}$
}

\begin{abstract}
Although a recent clinical trial (BEST TRIP) demonstrated no improvement in outcomes with invasive intracranial pressure (ICP) monitoring (ICPM) following severe traumatic brain injury (TBI), its generalizability has been called into question. In several global settings ICPM is not the standard of care and is used at the discretion of the attending neurosurgeon. Our objective was to determine the impact of ICPM on mortality and 6-month functional outcomes following severe TBI. The setting was a referral trauma center with 36 intensive care unit (ICU) beds and 300-600 TBI admissions per year. During a 2-year period data were prospectively entered into a severe TBI registry. Patients with severe TBI aged $>12$ years meeting Brain Trauma Foundation (BTF) criteria for ICPM were included in the study. Outcomes of interest were in-hospital mortality and poor 6-month functional outcome defined as Glasgow Outcome Scale (GOS) score of 3 or lower. A propensity score based analysis incorporating known predictors of outcome in TBI was utilized to examine the impact of ICPM on outcomes. Of 1345 patients meeting study criteria 497 (37\%) underwent ICPM. In-hospital mortality was 35\% (471/1345). Of 454 patients for whom 6-month outcome was available, 161 (35\%) suffered a poor functional outcome. Following propensity score analysis ICPM use was associated with an $8 \%(p=0.002)$ decrease in mortality but no significant effect $(p=0.2)$ on functional outcome. The use of ICPM following severe TBI was associated with decreased in-hospital mortality. Further clinical trials of ICPM in TBI may be warranted.
\end{abstract}

Key words: developing countries; Glasgow Outcome Scale; intracranial pressure; traumatic brain injury

\section{Introduction}

W ORLDWIDE, SEVERE TRAUMATIC BRAIN INJURY (TBI) is the most common cause of death and disability following injury, with motor vehicle-related injuries being the most common cause of TBI. ${ }^{1-3}$ The magnitude of the problem is particularly large in lowand middle-income nations, where more than $80 \%$ of the world's population lives. ${ }^{4} \mathrm{TBI}$ is the leading cause of disability in people under 40, resulting in severe disability for 150-200 people per million annually. ${ }^{5-8}$ Monitoring and control of intracranial pressure (ICP) in patients with severe TBI, defined by a Glasgow Coma Scale (GCS) score $\leq 8$, is an important part of Brain Trauma Foundation (BTF) guidelines, with the BTF recommending the use of invasive ICP monitoring (ICPM) in appropriate candidates with a goal ICP of $<20-25 \mathrm{~mm} \mathrm{Hg}$. ${ }^{9}$ Adherence to guidelines related to ICPM and management may have a significant impact on outcomes, with one study demonstrating an association between the appropriate use of ICPM and a decrease in mortality in New York State. ${ }^{10,11}$

The use of ICPM is associated with significant expense, resource utilization, and some risk, however, with the associated costs being particularly problematic in resource-constrained environments. In the absence of definitive evidence of benefit from clinical trials, the use of ICPM is therefore not typically considered the standard of care in many lower-income nations. Centers dealing with large volumes of TBI in these regions use a variety of alternative strategies to assess and manage ICP. Frequent clinical examinations and computed tomography (CT) imaging are sometimes used as surrogates for the assessment of ICP. A recent multi-center randomized clinical trial in South America (the BEST TRIP trial) demonstrated no benefit to the use of ICPM over a management protocol based on clinical monitoring and serial CT imaging. ${ }^{12}$ The generalizability of the study's findings have been called into question, particularly in view of the possible benefits in outcome demonstrated by the previously mentioned studies in the United States. ${ }^{10,11}$ It is possible that differences in therapeutic regimens used in the two arms (more hypertonic saline and hyperventilation in the ICPM arm and more barbiturate usage in the clinical/imaging arm), reflective of regional practice, may have had an impact on outcome in the BEST TRIP trial. It is also possible that strict adherence to the clinical monitoring and management protocol

\footnotetext{
${ }^{1}$ JPN Apex Trauma Center and Neurosciences Center, ${ }^{4}$ Department of Surgical Disciplines All India Institute of Medical Sciences, Ansari Nagar, India. ${ }^{2}$ Department of Surgery, ${ }^{3}$ Department of Biostatistics, ${ }^{5}$ Departments of Neurosurgery and Neurology, University of Michigan, Ann Arbor, Michigan.
} 
used in the control arm, in the setting of a clinical trial, may have diminished the additive benefit of ICPM. Because clinical practice outside the setting of a clinical trial is often subject to greater variation, it is possible that continuous invasive ICPM may provide additional value in the absence of the stringent monitoring requirements and protocol-driven therapy of a clinical trial.

Our objective therefore was to study the impact of ICPM on clinical outcomes-in-hospital mortality and 6-month functional outcome-in a setting reflective of contemporary clinical practice by querying a prospective severe TBI registry in a high-volume referral trauma center.

\section{Methods}

Approval of the respective Institutional Review Boards was obtained at both the University of Michigan (HUM00079298) and the All India Institute of Medical Sciences (AIIMS; IEC/NP-2752013) for this study. The Jai Prakash Narayan Apex Trauma Center (JPNATC) at AIIMS in New Delhi, is the apex referral center for TBI in New Delhi as well as for much of the rest of the country. It has 30 triage and 36 intensive care unit (ICU) beds, with 300-600 severe TBI admissions every year. This study was performed under the auspices of the University of Michigan/AIIMS research collaboration. All data collection and clinical care were performed at AIIMS, whereas analysis was performed at both institutions. The study period was June 2010-July 2012.

\section{Inclusion criteria}

1. Patients with severe TBI (GCS score $\leq 8$ )

2. Age $>12$ years. The age cutoff of 12 years was selected as being the threshold above which the clinical management of TBI and ICP was more consistent in the study setting, and also to maintain consistency with the selection criteria of the BEST TRIP trial.

3. BTF criteria for invasive ICPM met:

a. Abnormal CT scan or

b. Normal CT scan plus any two of the following

i. Age $>40$

ii. Systolic blood pressure $<90$

iii. Unilateral or bilateral posturing

\section{Exclusion criteria}

Death within 24 hours.

\section{Data source}

The data source was the AIIMS/JPNATC Severe TBI Registry, with prospective real-time electronic data capture supplemented by manual data entry and review. Manual data entry and review were performed by trained clinical research nurses with subsequent periodic review and validation by a physician. Six-month functional status as estimated by the Glasgow Outcome Scale (GOS), when available, is routinely entered into the registry. ${ }^{13}$ The 6-month GOS score was obtained through telephone interview conducted by trained research nurses and coordinators. A structured questionnaire was used during the interview with subsequent estimation of the GOS score from the responses obtained.

\section{Outcomes of interest}

The primary outcome of interest was in-hospital mortality, defined as death occurring from any cause during the patient's inpatient stay. This was selected as the primary outcome measure as being an objective measure available for all patients. The secondary outcome of interest was 6-month functional outcome in survivors of the in-hospital stay, with poor functional outcome defined as a GOS score of 3 or lower (including death following discharge from the in-hospital stay). An additional rationale for the use of the 6month telephonic assessment of GOS as a secondary, rather than primary, outcome measure was concern for a potential bias toward patients with better outcome being more accessible to follow-up.

Supplemental analyses were then performed for the outcome measures of 6-month mortality (including in-hospital deaths and death following discharge) and functional outcome in 6-month survivors. Poor functional outcome in this analysis was therefore a GOS score of 2 or 3 , in patients who were alive at 6-month follow-up.

\section{Intervention of interest and covariates}

The intervention of interest was the use of invasive ICPM during the patient's ICU admission. All patients included in this study met BTF criteria for ICPM; however, ICP monitors were placed variably at the discretion of the admitting neurosurgeon, as is standard practice at JPNATC. Intraparenchymal monitors (Codman $^{\mathrm{TM}}$, Depuy Synthes, West Chester, PA) were exclusively used for ICPM, with external ventricular drains (EVDs) reserved for the management of hydrocephalus. This practice, similar to the protocol of the BEST TRIP trial, reflects the perceived high rate of EVD infection in the study setting. ${ }^{12}$ Co-variates in the analysis were factors likely to influence the decision to place an ICP monitor, and included demographics, admission GCS score, pupillary reactivity, admission blood pressure, presence of extra-cerebral traumatic injury (separated into acute spinal cord injury and other system injury), and specific abnormalities on CT scan. Of note, many of these variables are also known predictors of outcome following TBI and therefore potential confounders. ${ }^{14}$ The specific abnormalities on CT, entered into the model as separate variables, were: presence of basal cisternal effacement, midline shift in millimeters, presence of traumatic subarachnoid hemorrhage (tSAH), presence of epidural hematoma $(\mathrm{EDH})$, presence of subdural hematoma (SDH), and presence of intraparenchymal contusion. Decompressive craniectomy (DC) was performed at the discretion of the attending neurosurgeon either pre-emptively, when, in the judgment of the neurosurgeon the patient was at high risk for the development of intracranial hypertension based on review of clinical and radiographical data, or as a rescue measure for intracranial hypertension refractory to medical management. Early DC (eDC) was defined in our study as DC performed pre-emptively within $48 \mathrm{~h}$ of injury on the basis of assessment of the patient's clinical examination and radiographical features (primarily midline shift and basal cisternal effacement) and a consequent determination of a high risk for refractory intracranial hypertension by the attending neurosurgeon. Patients undergoing eDC subsequently underwent invasive ICP monitor placement at the discretion of the attending neurosurgeon. Additional analyses were performed to account for a potential confounding effect of eDC on outcomes, because patients who underwent eDC may have been less likely to undergo subsequent ICPM and the interpretation and response to ICP values following eDC may differ compared with patients with a "closed skull." First, a separate propensity score analysis was performed with inclusion of eDC as a covariate in the calculation of the propensity score. Second, a subgroup analysis was performed for the same outcome measures, excluding all patients who underwent eDC.

\section{Management principles}

Although no standardized TBI protocol was used, the following broad principles of management were in effect. Patients with severe TBI were admitted to the neurointensive care unit (neuroICU) of JPNATC. Care of patients with TBI in the neuroICU was provided by neurosurgical housestaff supervised by senior neurosurgical faculty and neurointensivists. Patients with GCS score $\leq 8$ were intubated, with tracheostomy performed when the need for airway protection or mechanical ventilation was anticipated for $>3$ weeks. 
Normal saline was used to maintain euvolemia. Additionally, vasopressors were utilized as required to maintain cerebral perfusion pressure (CPP) $>60 \mathrm{~mm} \mathrm{Hg}$ in patients with ICP monitors and mean arterial pressure (MAP) $>80-90 \mathrm{~mm} \mathrm{Hg}$ in patients without ICP monitors. When ICPM was used, the goal ICP was $<20 \mathrm{~mm} \mathrm{Hg}$. When an ICP monitor was not placed, the use of measures directed at management of cerebral edema and raised ICP was based on review of the patient's neurological condition and serial CT imaging, without a standardized clinical management protocol. Mannitol was used for osmotherapy with scheduled (rather than asneeded) dosing regardless of whether ICPM was used, with doses held for hypotension, hypovolemia, renal failure, serum sodium $>155$ or serum osmolality $>320$. Partial pressure of carbon dioxide $\left(\mathrm{PCO}_{2}\right)$ was generally maintained between 30 and $40 \mathrm{~mm} \mathrm{Hg}$ with therapeutic hyperventilation to $\mathrm{PCO}_{2}<30 \mathrm{~mm} \mathrm{Hg}$ used only for limited duration as a rescue measure in the setting of cerebral herniation or severe neurological deterioration caused by raised ICP. Standard measures to maintain normothermia (temperature $<38^{\circ} \mathrm{C}$ ), including scheduled acetaminophen, evaporative surface cooling with water spray and bedside fan, ice packs, and cooling blankets, were utilized; however, hypothermia was not used. DC was performed as described in the section above.

\section{Statistical analysis}

Overview of the propensity-score based analysis. Multiple analyses were carried out to evaluate the effect of ICPM on each of the outcomes: death and poor functional outcome at 6 months. For each analysis, the main idea was to subset the patients such that the probability of treatment was approximately equal within each subgroup; in which case treatment can be viewed as having been (approximately) randomized. Treatment-and-subgroup-specific fractions of patients experiencing the outcome (e.g., death) were calculated then combined. Propensity score analysis is useful in estimating the effect of an intervention (ICPM) in a retrospective observational study while adjusting for the covariates, or baseline variables, that predict receiving the intervention. ${ }^{15,16}$ Propensity score analysis decreases the bias due to confounding variables that can occur when the intervention effect is estimated by simply comparing outcomes among patients receiving the intervention with those who did not.

Estimation of propensity score. Logistic regression modeling was first used to predict the probability of receiving ICPM for each patient (i.e., the propensity score) as a function of several potential confounding variables: age, gender, time from injury to admission, admission GCS score, pupillary reactivity, admission blood pressure, presence of associated spinal injury, presence of associated non-neurological traumatic injury, presence of basal cisternal effacement, midline shift in millimeters, presence of tSAH, presence of SDH, presence of $\mathrm{EDH}$, presence of non-evacuated hematoma, and presence of parenchymal contusion. ${ }^{14}$ In addition, performance of eDC was included as a covariate in the analysis of the impact of ICPM as a potential confounder, as described earlier. A Cindex was calculated to evaluate the ability of the logistic regression model to differentiate between patients pairs of patients discordant with respect to use of ICPM. A C-index of $>0.7$ was considered adequate, for use of the model to predict ICPM use.

Propensity-score adjusted comparison of ICPM versus no-ICPM. The predicted probabilities (propensity scores) were then recoded into deciles, and a new factor was computed indicating the decile to which each patient was assigned based on their propensity score. The treatment-specific probability of dying was then estimated within each decile as the fraction of subjects who died. These decile-specific fractions were then averaged for each treatment and compared. The null hypothesis of equal death probability across treatments (i.e., ICPM versus no-ICPM) was then tested using the Z-test based on the standard normal distribution. The procedure outlined in the preceding paragraph was then repeated for the outcome poor-functional status (GOS score $\leq 3$ ). The primary analysis of functional outcome included only patients with a 6-month measurement available, and excluded patients who died in the hospital. The response variate in this case should be interpreted as pertaining to the probability of poor functional outcomes among surviving patients (i.e., those discharged alive). Supplemental analyses were then performed for the outcome measures of 6-month mortality (including in-hospital deaths and deaths following discharge) and functional outcome in 6-month survivors. Poor functional outcome in this analysis was therefore a GOS score of 2 or 3 , in patients who were alive at 6-month follow-up.

All statistical analyses were carried out using SAS version 9.3 (SAS Institute; Cary, NC).

\section{Results}

There were 1530 admissions for severe TBI during the period of the study. Of these, 1345 met criteria for inclusion in the study. The median age was 32 years (interquartile range [IQR] 25-45 years), $89 \%$ of whom were male. The primary cause of injury was road traffic accident $(71 \%)$ with falls $(20 \%)$ being the next common cause. The distribution of variables and outcomes in patients with and without use of ICPM is shown in Table 1.

\section{Use of ICPM and decompressive craniectomy and outcomes}

Overall, 497 (37\%) patients underwent ICPM. Median ICP at time of monitor placement was $12 \mathrm{~mm} \mathrm{Hg}$ (IQR $8-18 \mathrm{~mm} \mathrm{Hg}$ ). The highest recorded ICP was $>20 \mathrm{~mm} \mathrm{Hg}$ in 75 of 497 (15\%) patients. A total of 589 (44\%) patients underwent DC, whereas 491 (37\%) underwent eDC. In-hospital mortality was $35 \%$ (471/1345). Six-month outcome assessment was available in 52\% (454/874) of survivors. Of these, $35 \%(161 / 454)$ were considered to have a poor functional outcome (GOS score 1, 2, or 3). The GOS breakup among patients with follow-up was: GOS $1=92(20 \%)$, GOS $2=18(4 \%)$, GOS $3=55(12 \%)$, GOS $4=92(20 \%)$, and GOS $5=197(43 \%)$.

\section{Propensity score analysis}

The C-index for the logistic regression model used to predict ICPM was 0.74 . For the propensity score analysis to evaluate ICPM in the primary analysis, the deciles used to group patients by propensity score were computed twice: once for the analysis of inhospital death, and a second time for the analysis of 6-month outcomes (with the latter being restricted to patients who survived 6 months and had a 6-month outcomes measure available). Results are summarized in Table 2A. ICPM was associated with a significant $(p=0.001)$ decrease in the probability of in-hospital death of approximately 9\%. ICPM did not have a significant effect ( $p=$ 0.46 ) on the probability of poor function at 6 months. Including early DC in the calculation of the propensity score, results were virtually unchanged (Table 2B). In the subgroup analysis with exclusion of patients who underwent eDC $(n=854)$, ICPM was again associated with a $9 \%$ reduction in in-hospital mortality $(p=0.002)$, whereas the difference in 6-month functional outcome did not attain the threshold for statistical significance $(p=0.08)$.

Results of the supplemental propensity score analysis with the outcome measures of 6-month mortality and poor functional outcome in 6-month survivors (GOS score $=2$ or 3 in patients alive at 6 months) are depicted in Table 3. Use of ICPM was associated with a significant $6 \%$ reduction $(p=0.03)$ in 6-month mortality and no 
Table 1. Distribution of Variables in Patients with and without Use of ICPM

\begin{tabular}{|c|c|c|c|}
\hline Variable & $\begin{array}{c}\text { ICPM not used } \\
\mathrm{N}=848\end{array}$ & $\begin{array}{c}\text { ICPM used } \\
\mathrm{N}=497\end{array}$ & $\mathrm{P}$ value \\
\hline Age in years, median (IQR) & $32(25-45)$ & $31(25-42)$ & 0.22 \\
\hline Female gender & $92(11 \%)$ & $59(12 \%)$ & 0.63 \\
\hline Hours from injury to admission, median (IQR) & $3.46(11.3)$ & $3.08(10.9)$ & 0.26 \\
\hline Admission GCS, median (IQR) & $6(4-7)$ & $7(6-7)$ & $<0.0001$ \\
\hline Pupillary reactivity absent & & & $<0.0001$ \\
\hline One side & $89(10 \%)$ & $44(9 \%)$ & \\
\hline Both sides & $183(22 \%)$ & $36(7 \%)$ & \\
\hline Admission systolic blood pressure in $\mathrm{mm} \mathrm{Hg}$, median (IQR) & $125(112-140)$ & $124(112-136)$ & 0.74 \\
\hline Admission $\mathrm{PO}_{2}$ in $\mathrm{mm} \mathrm{Hg}$, median (IQR) & $185(131-242)$ & $200(142-251)$ & 0.014 \\
\hline Associated spine injury present & $51(6 \%)$ & $32(6 \%)$ & 0.84 \\
\hline Other systemic trauma present & $212(25 \%)$ & $128(26 \%)$ & 0.81 \\
\hline CT midline shift in mm (IQR) & $2.9(0-8)$ & $0(0-3)$ & $<0.0001$ \\
\hline Basal cisternal effacement on CT & $166(20 \%)$ & $61(12 \%)$ & 0.0007 \\
\hline Traumatic subarachnoid hemorrhage present & $301(35 \%)$ & $202(41 \%)$ & 0.068 \\
\hline Extradural hematoma present & $189(22 \%)$ & $54(11 \%)$ & $<0.0001$ \\
\hline Subdural hematoma present & $433(51 \%)$ & $192(39 \%)$ & $<0.0001$ \\
\hline Intraparenchymal contusion present & $498(59 \%)$ & $347(70 \%)$ & 0.0001 \\
\hline Non-evacuated hematoma present & $127(15 \%)$ & $114(23 \%)$ & 0.0002 \\
\hline Early decompressive craniectomy performed & $374(44 \%)$ & $117(23 \%)$ & $<0.0001$ \\
\hline In-hospital death & $353(42 \%)$ & $118(24 \%)$ & $<0.0001$ \\
\hline Poor 6-month outcome & $94(11 \%)$ & $66(13 \%)$ & 0.98 \\
\hline
\end{tabular}

CT, computed tomography; GCS, Glasgow Coma Scale; ICPM, intracranial pressure monitoring; IQR, interquartile range; $\mathrm{PO}_{2}$, partial pressure of oxygen.

significant difference in poor functional outcome in 6-month survivors $(p=0.17)$. In the subgroup analysis with exclusion of patients who underwent eDC (Table 3B, $n=854)$, ICPM was again associated with a $7 \%$ reduction in 6-month mortality $(p=0.03)$, whereas the difference in poor functional outcome in 6-month survivors did not attain the threshold for statistical significance $(p=0.08)$.

\section{Discussion}

Our study examined the impact of invasive ICPM on outcomes following severe TBI using a propensity score based analysis. The use of ICPM was associated with a significant reduction in inhospital mortality (and 6-month mortality) with no significant difference in 6-month functional outcomes, whether defined as a GOS score of 1-3 at 6 months in survivors of the in-hospital stay or a GOS score of 2-3 in 6-month survivors. Strengths of our study include a relatively large sample size $(n=1345)$, availability of 6-month functional outcomes, availability for comparative analysis of a relatively large group of control patients who did not undergo ICPM despite meeting BTF criteria (because ICPM was not considered standard of care in the study setting), validated prospective data entry into the TBI registry, and a study population comparable to that of the BEST TRIP trial. Our findings are consistent with those of studies of the impact of ICPM from New York State and Los Angeles, both of which revealed an association with lower in-hospital mortality, but did not study 6-month functional outcomes. ${ }^{10,17}$ Similar to our study, these studies examined existing practice outside the context of a clinical trial, albeit in a different practice setting. The significant reduction in mortality seen in our study, as well as the aforementioned studies, may therefore suggest that the continued use of ICPM in appropriately selected patients is warranted.

The only large randomized controlled trial of invasive ICPM, the BEST TRIP trial, did not, however, demonstrate an improvement in outcomes with the use of invasive ICPM, although it must be noted that the ICPM group in the BEST TRIP trial did have a lower 14day mortality ( $21 \%$ vs. $30 \%)$, a difference that did not reach statistical significance $(p=0.18) .{ }^{12}$ The BEST TRIP trial, a clinical trial with likely robust internal validity, has been subject to debate with regards to the external validity of its findings. ${ }^{18,19}$ Potentially the most important difference may be between the controlled milieu of a clinical trial, particularly one such as BEST TRIP with a high degree of reported adherence to the study protocol, and routine clinical practice, where the intensity and consistency of clinical neurological monitoring as well as management may be subject to greater variation. This may be particularly true at large teaching institutions where a substantial proportion of care is provided by clinicians in training, in understaffed clinical environments and settings in which the bedside nurse in the ICU may not routinely care for a large volume of neurosurgical patients. Clinical signs of deterioration and impending herniation, such as a downward drift in the GCS score or mild pupillary asymmetry, may therefore be overlooked, whereas a numerical value such as the ICP, when clearly above a target threshold, may trigger a more expeditious response. Other important differences exist between our study and the BEST TRIP trial. Barbiturate coma and hypertonic saline were not used in our study setting, whereas both were used in the BEST TRIP trial with a statistically significant difference in utilization between the treatment and control arms. ${ }^{12}$ Where ICPM is not used, it is likely essential to adhere to a rigorous protocol of frequent clinical and imaging re-assessment and proactive management, similar to the one used in BEST TRIP.

Similar to BEST TRIP, the use of ICPM was not associated with an improvement in six-month functional outcome in our study. This, however, does not necessarily imply more lives saved at the cost of severe disability and an increased burden to society. This is because in-hospital mortality was the more reliable and robust outcome measure in our study, available for all patients, whereas 
Table 2. Results of Propensity Score Analysis: IMPACT OF ICPM ON IN-Hospital MORTALITy and 6-Month Functional Outcome in Survivors of THE In-Hospital Stay

\begin{tabular}{|c|c|c|}
\hline Group & $\begin{array}{l}\text { Propensity-score } \\
\text { adjusted } \\
\text { probability of } \\
\text { in-hospital death }\end{array}$ & $\begin{array}{c}\text { Propensity-score } \\
\text { adjusted } \\
\text { probability of poor } \\
\text { function at } 6 \text { months }\end{array}$ \\
\hline
\end{tabular}

2A. Impact of ICPM on in-hospital death and 6-month functional outcome. All patients included.

\begin{tabular}{lcc}
\hline ICPM & 0.247 & 0.361 \\
No ICPM & 0.320 & 0.323 \\
Difference: ICPM & -0.0732 & 0.0387 \\
$\quad$ minus no-ICPM & & \\
SE (difference) & 0.0248 & 0.0529 \\
Z-score: difference/SE & -2.949 & 0.732 \\
$P$ value & $P=0.003$ & $P=0.464$ \\
\hline
\end{tabular}

2B. Impact of ICPM on in-hospital death and 6-month functional outcome with eDC included as covariate for propensity score. All patients included.

\begin{tabular}{lcc}
\hline ICPM & 0.253 & 0.357 \\
No ICPM & 0.317 & 0.323 \\
Difference: ICPM & -0.0638 & 0.0348 \\
$\quad$ minus no-ICPM & & \\
SE (difference) & 0.0251 & 0.0525 \\
Z-score: difference/SE & -2.544 & 0.663 \\
$P$ value & $P=0.011$ & $P=0.507$ \\
\hline
\end{tabular}

2C. Impact of ICPM on in-hospital death and 6-month functional outcome: subgroup excluding patients who underwent $e D C$

\begin{tabular}{lcc}
\hline ICPM & 0.182 & 0.310 \\
No ICPM & 0.270 & 0.223 \\
Difference: ICPM & -0.0879 & 0.0866 \\
$\quad$ minus no-ICPM & & \\
SE (difference) & 0.0282 & 0.0492 \\
$Z$-score: difference/SE & -3.119 & 1.758 \\
$P$ value & $P=0.002$ & $P=0.079$ \\
\hline
\end{tabular}

Poor 6-month functional outcome in this analysis was defined as GOS score $\leq 3$ (including patients who died following discharge from the hospital).

eDC, early decompressive craniectomy; ICPM, intracranial pressure monitoring; SE, standard error.

six-month outcome was available in about half of survivors. As described earlier, we cannot exclude a potential bias toward patients with better functional outcomes being more accessible to follow-up, thereby confounding the analysis of the impact of the intervention on outcomes. A biological basis to the absence of an impact of ICPM on functional outcomes despite a difference in mortality certainly remains a possibility, because averting death related to intracranial hypertension may not alter the substantial underlying neurological injury and subsequent poor functional outcome. It is also possible that the limited availability of highquality rehabilitation services in the post-discharge study setting (again, similar to BEST TRIP) blunted any benefit in functional outcome among survivors-that is, although the extent of secondary cerebral injury may have been minimized with the intervention, patients were unable to translate this benefit into greater independence, in the absence of appropriate physical, occupational,
Table 3. Results of Propensity Score Analysis: ImPaCt of ICPM on 6-Month Mortality (Dead at 6 Months, Including Death during In-Hospital Stay and Deaths following Discharge) and 6-Month Functional Outcome in Patients Alive at 6 Months

\begin{tabular}{|c|c|c|}
\hline Group & $\begin{array}{c}\text { Propensity-score } \\
\text { adjusted probability } \\
\text { of death within } \\
6 \text { months }\end{array}$ & $\begin{array}{l}\text { Propensity-score } \\
\text { adjusted probability } \\
\text { of poor function } \\
\text { at } 6 \text { months among } \\
\text { 6-month survivors }\end{array}$ \\
\hline
\end{tabular}

3A. Impact of ICPM on 6-month death and functional outcome among 6-month survivors. All patients included.

\begin{tabular}{|c|c|c|}
\hline ICPM & 0.381 & 0.213 \\
\hline No ICPM & 0.373 & 0.147 \\
\hline $\begin{array}{l}\text { Difference: ICPM } \\
\text { minus no-ICPM }\end{array}$ & -0.0554 & 0.0659 \\
\hline SE (difference) & 0.0262 & 0.0478 \\
\hline Z-score: difference/SE & -2.118 & 1.379 \\
\hline$P$ value & $P=0.034$ & $P=0.168$ \\
\hline \multicolumn{3}{|c|}{$\begin{array}{l}\text { 3B. Impact of ICPM on 6-month mortality and functional } \\
\text { outcome among 6-month survivors: subgroup excluding } \\
\text { patients who underwent eDC }\end{array}$} \\
\hline ICPM & 0.243 & 0.179 \\
\hline No ICPM & 0.309 & 0.105 \\
\hline $\begin{array}{l}\text { Difference: ICPM } \\
\text { minus no-ICPM }\end{array}$ & -0.0659 & 0.0741 \\
\hline SE (difference) & 0.0303 & 0.0417 \\
\hline Z-score: difference/SE & -2.179 & 1.777 \\
\hline$P$ value & $P=0.029$ & $P=0.076$ \\
\hline
\end{tabular}

Poor 6-month functional outcome in this analysis was defined as a GOS score of 2 or 3 in patients alive at 6 months.

eDC, early decompressive craniectomy; ICPM, intracranial pressure monitoring; SE, standard error.

speech/language, and other focused rehabilitative therapy. For all these reasons, no definitive conclusion can be drawn from our study regarding the impact of ICPM on functional outcomes.

The relatively high usage (37\%) of eDC in our study partly reflects the desire to minimize the subsequent need for ICP-directed resources, and, especially, ICU length of stay. In fact, the performance of DC in the DECRA trial was associated with a much lower need for ICP-directed medical treatment and fewer ICU days. ${ }^{20}$ The relatively low incidence of intracranial hypertension (15\%) among patients undergoing ICPM in our study may reflect the aggressive use of eDC. The aggressive use of eDC may therefore dilute the need for and subsequent impact of ICPM on outcomes. Conversely, because the use of DC was associated with worse functional outcomes in the DECRA trial, the aggressive use of eDC may confound functional outcomes. It is unlikely that $\mathrm{eDC}$ was a significant confounder, however, because additional analyses with inclusion of $\mathrm{eDC}$ as a co-variate in the calculation of the propensity score, as well as subgroup analysis excluding all patients who underwent eDC, yielded similar results.

The major limitation of our study is its retrospective nature, although data were collected prospectively. The propensity score analysis is likely to have mitigated any major imbalances in baseline variables, however. Although the management of patients with ICPM was largely guided by BTF guidelines and the principles outlined in the "Management principles" section above, there was no mandatory standardized protocol in effect, as was employed 
in the BEST TRIP trial; our study was, instead, a pragmatic evaluation of existing clinical practice. Six-month outcome was available for only about half of survivors and as previously mentioned a bias toward patients with better outcomes being more likely to respond to telephone outreach is possible. The aggressive use of eDC may have mitigated any potential benefit from the use of ICPM to direct therapy. We were unable to analyze potential disparities in resource utilization and costs between the intervention and comparator groups. Finally, despite our inclusion of major co-variates proven to predict poor outcome following TBI in the logistic regression model used to calculate the propensity score, the influence of unmeasured confounders cannot be entirely excluded.

In conclusion, the use of invasive ICPM in patients with severe TBI was associated with decreased in-hospital mortality. Continued utilization of ICPM in appropriately selected patients, as well as further clinical trials in settings where the use of invasive ICPM is not the standard of care, may therefore be warranted.

\section{Acknowledgments}

Funding for this study was in part provided by the India Collaborative Fund from the Department of Surgery at the University of Michigan.

\section{Author Disclosure Statement}

No competing financial interests exist.

\section{References}

1. Murray, C.J., and Lopez, A.D. (1997). Global mortality, disability, and the contribution of risk factors: Global Burden of Disease Study. Lancet 349, 1436-1442.

2. Bruns, J. Jr., and Hauser, W.A. (2003). The epidemiology of traumatic brain injury: a review. Epilepsia 44, (Suppl 10), 2-10.

3. Dandona, R., Kumar, G.A., Ameer, M.A., Ahmed, G.M., and Dandona, L. (2008). Incidence and burden of road traffic injuries in urban India. Inj. Prev. 14, 354-359.

4. De Silva, M.J., Roberts, I., Perel, P., Edwards, P., Kenward, M.G., Fernandes, J., Shakur, H., Patel, V.; CRASH Trial Collaborators. (2009). Patient outcome after traumatic brain injury in high-, middleand low-income countries: analysis of data on 8927 patients in 46 countries. Int. J. Epidemiol. 38, 452-458.

5. Langlois, J.A., Rutland-Brown, W., and Wald, M.M. (2006). The epidemiology and impact of traumatic brain injury: a brief overview. J. Head Trauma Rehabil. 21, 375-378.

6. Gururaj, G. Road traffic deaths, injuries and disabilities in India: current scenario. (2008). Natl. Med. J. India 21, 14-20.

7. Ameratunga, S., Hijar, M., and Norton, R. (2006). Road-traffic injuries: confronting disparities to address a global-health problem. Lancet 367, 1533-1540.

8. Gururaj, G. (2002). Epidemiology of traumatic brain injuries: Indian scenario. Neurol. Res. 24, 24-28.

9. Brain Trauma Foundation; American Association of Neurological Surgeons; Congress of Neurological Surgeons; Joint Section on
Neurotrauma and Critical Care, AANS/CNS. (2007). Guidelines for the management of severe traumatic brain injury. VI. Indications for intracranial pressure monitoring. J. Neurotrauma 24 (Suppl 1), S37S44.

10. Farahvar, A., Gerber, L.M., Chiu, Y.L., Carney, N., Härtl, R., and Ghajar, J. (2012). Increased mortality in patients with severe traumatic brain injury treated without intracranial pressure monitoring. J. Neurosurg. 117, 729-34.

11. Gerber, L.M., Chiu, Y.L., Carney, N., Härtl, R., Ghajar, J. (2013). Marked reduction in mortality in patients with severe traumatic brain injury. J. Neurosurg. 119, 1583-1590.

12. Chesnut, R.M., Temkin, N., Carney, N., Dikmen, S., Rondina, C., Videtta, W., Petroni, G., Lujan, S., Pridgeon, J., Barber, J., Machamer, J., Chaddock, K., Celix, J.M., Cherner, M., and Hendrix, T. (2012). A trial of intracranial-pressure monitoring in traumatic brain injury. N. Engl. J. Med. 367, 2471-2481.

13. Jennett, B., and Bond, M. (1975). Assessment of outcome after severe brain damage. Lancet 1, 480-484.

14. Chesnut, R.M., Ghajar, J., Maas, A.I., Marion, D.W., Servadei, F., Teasdale, G.M., Unterberg, A., Von Holst, H., and Walters, B.C. (2000). Early indicators of prognosis in severe traumatic brain injury. J. Neurotrauma 17, 557-627.

15. Rosenbaum, P.R., and Rubin, D.B. (1983). The central role of the propensity score in observational studies for causal effects. Biometrika 70, 41-55.

16. Heinze G., and Jüni, P. (2011). An overview of the objectives of and the approaches to propensity score analyses. Eur. Heart J. 32, 1704-1708.

17. Dawes, A.J., Sacks, G.D., Cryer, H.G., Gruen, J.P., Preston, C., Gorospe, D., Cohen, M., McArthur, D.L., Russell, M.M., MaggardGibbons, M., Ko, C.Y.; Los Angeles County Trauma Consortium. (2015). Intracranial pressure monitoring and inpatient mortality in severe traumatic brain injury: a propensity score-matched analysis. J. Trauma Acute Care Surg. 78, 492-502.

18. Sahuquillo, J., Martínez-Ricarte, F., and Poca, M.A. (2013). Decompressive craniectomy in traumatic brain injury after the DECRA trial. Where do we stand? Curr. Opin. Crit. Care 19, 101-106.

19. Chesnut, R.M. (2013). Intracranial pressure monitoring: headstone or a new head start. The BEST TRIP trial in perspective. Intensive Care Med. 39, 771-774.

20. Cooper, D.J., Rosenfeld, J.V., Murray, L., Arabi, Y.M., Davies, A.R., D’Urso, P., Kossmann, T., Ponsford, J., Seppelt, I., Reilly, P., Wolfe, R.; DECRA Trial Investigators; Australian and New Zealand Intensive Care Society Clinical Trials Group. (2011). Decompressive craniectomy in diffuse traumatic brain injury. New Engl. J. Med. 364, 1493-502.

Address correspondence to:
Venkatakrishna Rajajee, MD
Departments of Neurosurgery and Neurology
University of Michigan
3552 Taubman Health Care Center
1500 E. Medical Center Drive
Ann Arbor, MI 48109
E-mail: vrajajee@yahoo.com

Address correspondence to: rosurgery and Neurology an Health Care Center er Drive

E-mail:vrajajee@yahoo.com 


\section{This article has been cited by:}

1. Nithya Kannan, Alex Quistberg, Jin Wang, Jonathan I. Groner, Richard B. Mink, Mark S. Wainwright, Michael J. Bell, Christopher C. Giza, Douglas F. Zatzick, Richard G. Ellenbogen, Linda Ng Boyle, Pamela H. Mitchell, Monica S. Vavilala. 2017. Frequency of and factors associated with emergency department intracranial pressure monitor placement in severe paediatric traumatic brain injury. Brain Injury 31:13-14, 1745-1752. [Crossref]

2. Alice Piccinini, Meghan Lewis, Elizabeth Benjamin, Alberto Aiolfi, Kenji Inaba, Demetrios Demetriades. 2017. Intracranial pressure monitoring in severe traumatic brain injuries: a closer look at level 1 trauma centers in the United States. Injury 48:9, 1944-1950. [Crossref]

3. Alberto Aiolfi, Elizabeth Benjamin, Desmond Khor, Kenji Inaba, Lydia Lam, Demetrios Demetriades. 2017. Brain Trauma Foundation Guidelines for Intracranial Pressure Monitoring: Compliance and Effect on Outcome. World Journal of Surgery 41:6, 1543-1549. [Crossref]

4. Nino Stocchetti, Tommaso Zoerle, Marco Carbonara. 2017. Intracranial pressure management in patients with traumatic brain injury. Current Opinion in Critical Care 23:2, 110-114. [Crossref] 